\title{
Time Crystals at Last?
}

1. Observation of Time-Crystalline Eigenstate Order on a Quantum Processor

Authors: Google Quantum AI and collaborators

arXiv:2107.13571

2. Observation of a many-body-localized discrete time crystal with a programmable spin-based quantum simulator

Authors: J. Randall et al.

arXiv:2107.00736

\section{Phase Structure of Driven Quantum Systems}

Authors: V. Khemani, A. Lazarides, R. Moessner, and S. L. Sondhi

Phys. Rev. Lett., 116, 250401 (2016)

\section{Recommended with a Commentary by Daniel Arovas, University of California, San Diego}

The intriguing possibility of spontaneous breaking of time translation symmetry, resulting in a novel phase of matter called a time crystal, was raised by Wilczek in 2012. Alas, a particularly natural definition of such a phase, in which the spacetime correlator $\langle\phi(\boldsymbol{x}, t) \phi(\mathbf{0}, 0)\rangle$ of an order parameter field tends to a nonconstant periodic function $C(t)$ as $|\boldsymbol{x}| \rightarrow \infty$, was proven to be impossible by Watanabe and Oshikawa, whether $C(t)$ is computed in a ground state or in a thermal ensemble [1]. In 2016, however, it was argued that a version of timecrystalline order does exist in many-body localized (MBL) one-dimensional Floquet-driven systems, where the Hamiltonian $H(t)=H(t+T)$ is a periodic function of time. In such systems, observables exhibit a doubled period thus breaking a discrete time translational symmetry $[2,3,4] .{ }^{1}$ While this discrete time crystal may seem to be somewhat impoverished from the symmetry viewpoint, from a quantum statistical mechanical viewpoint it is remarkable in that it also exhibits the phenomena of eigenstate order and absolute stability (described below). Despite previous work on the topic, it was only very recently that groups at Google [6] and at Delft University of Technology [7] have obtained substantial evidence for the existence of such a discrete time crystal (DTC) phase, using quantum simulators.

\footnotetext{
${ }^{1}$ For an earlier JCCM contribution highlighting the work of ref. [3], see [5].
} 
Subharmonic generation, here at frequency $\frac{1}{2} \Omega$ where $\Omega=2 \pi / T$ is the drive frequency, has of course been appreciated in various contexts, and for centuries. However, its manifestation in non-dissipative many-body quantum systems heretofore has no precedent; this is what is new about the MBL-DTC phase. The work of Khemani et al. [2] identified, for the first time, several distinct phases of matter in a nonequilibrium MBL-Floquet setting, among them the DTC. This is possible owing to the notion of eigenstate order, whereby individual many-body eigenstates of the dynamics can exhibit symmetry-breaking or topological order, even where the strictures of equilibrium thermodynamics would forbid such a state of affairs [9]. In the MBL-DTC phase, the eigenstate ordering is spatio-temporal in nature. The Floquet eigenstates resemble Schrödinger cat states $\left|\Psi_{\nu, \pm}\right\rangle=2^{-1 / 2}(|\nu\rangle \pm P|\nu\rangle)$, where each $|\nu\rangle$ has a spatial spin-glass character, and $P$ flips all the spins. Under the unitary evolution $U_{\mathrm{F}}$ corresponding to one drive cycle, a relative phase accumulation of $\pi$ accrues over one period, so that $U_{\mathrm{F}}\left|\Psi_{\nu, \pm}\right\rangle=e^{-i \theta_{\nu}}\left\{\begin{array}{c}+1 \\ -1\end{array}\right\}\left|\Psi_{\nu, \pm}\right\rangle$, and it takes general linear combination of the cats two periods to return to itself. Thus, under generic initial conditions, period doubling arises. Remarkably, this feature is robust with respect to all perturbations with the same period $T[3,4]$, which the authors of ref. [4] refer to as 'absolute stability'. This is distinct from the conventional ordered phase of, say, an Ising magnet, where applying an external field which breaks the Ising symmetry destroys the distinction between ferromagnet and paramagnet, replacing the phase transition with a crossover.

In Floquet systems, the (unitary) evolution operator is the time ordered exponential $U(t, 0)=\mathcal{T} \exp \left\{-i \hbar^{-1} \int_{0}^{t} d t H(t)\right\}$ and the Floquet unitary $U_{\mathrm{F}}=U(T, 0)$ implements evolution over a single period. The eigenvalues of $U_{\mathrm{F}}$ are phases $\exp \left(-i \mathcal{E}_{\alpha} T / \hbar\right)$, where the $\left\{\mathcal{E}_{\alpha}\right\}$ are the quasienergies, each defined modulo $\hbar \Omega$. Like crystal momentum in a periodic potential being defined modulo reciprocal lattice vectors, each quasienergy is defined modulo $\hbar \Omega$. One might think that one can always write $U_{\mathrm{F}}=e^{-i H_{\mathrm{F}} T / \hbar}$ with $H_{\mathrm{F}}$ a 'Floquet Hamiltonian', but taking the logarithm of $U_{\mathrm{F}}$ is tricky business, and generically this results in Hamiltonians which are either very long-ranged or involve arbitrarily many-body interactions.

A driven system can exchange energy with its driver. From the second law of thermodynamics, we therefore expect that the evolution will be toward a state of maximum entropy, and since energy is not conserved, this means heating to $T=\infty$, where all quantum states are equally likely. This is an ergodic phase, and according to the eigenstate thermalization hypothesis (ETH) we expect all the eigenstates of $U_{\mathrm{F}}$ will encode $T=\infty$ properties, which is to say a complete absence of correlations. This is called the Floquet-ETH (thermal) phase, and it is the only ergodic phase of periodically driven systems in the thermodynamic limit.

To avoid the fate of heat death, a thermodynamically large number of conserved quantities is needed. If, for example, $H_{\mathrm{F}}$ is a free fermion Hamiltonian, then the occupation numbers of all the fermion modes are conserved - a highly nongeneric situation which is not stable to generic perturbations. By contrast, when the eigenstates of $U_{\mathrm{F}}$ are many-body localized [8], a robust Floquet-MBL phase may arise. In this case, the conserved quantities are the so-called $\ell$-bit (for "localized bit") Pauli operators $\tau_{n}^{z}$, which can be regarded as 'dressed' versions of the Pauli operators $Z_{n}$. Unlike the Floquet-ETH phase, which is unique, it was found in ref. [2] that there can be several Floquet-MBL phases, one of which is the discrete 

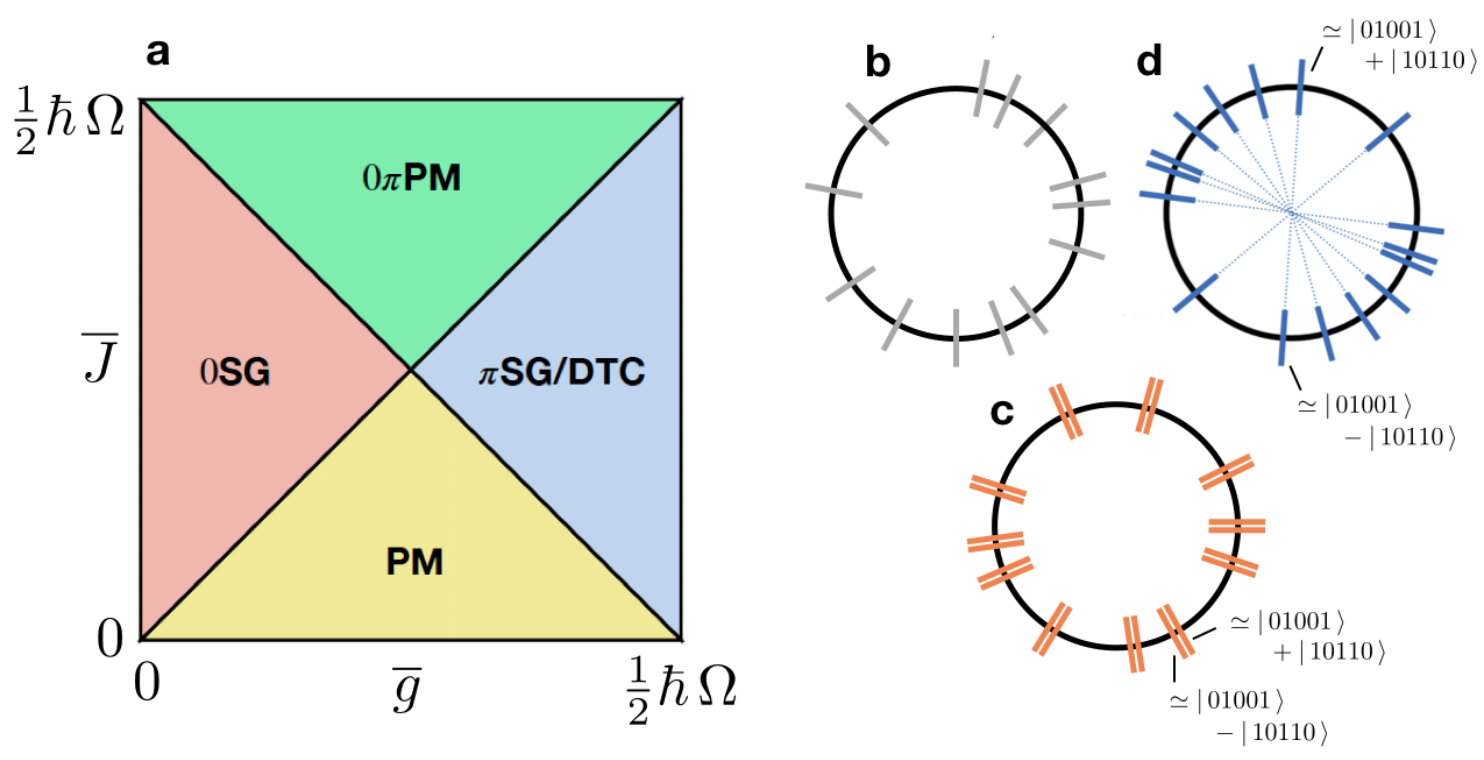

Figure 1: (a) Phase diagram for the noninteracting model of eqn. 1 , with $g_{n}=\bar{g}$ and $J_{n}=\bar{J}$ for all $n$ (or their disorder averages). Quasienergy spectra sketches shown for (b) FloquetETH phase (no cat states), (c) 0SG phase, and (d) $\pi \mathrm{SG} / \mathrm{DTC}$ phase. With disorder and interactions as in eqn. 1, each region typifies a Floquet-MBL phase. Adapted from [2, 6].

time crystal.

The model considered by Khemani, Lazarides, Moessner, and Sondhi (KLMS) [2] is one with binary drive, for which

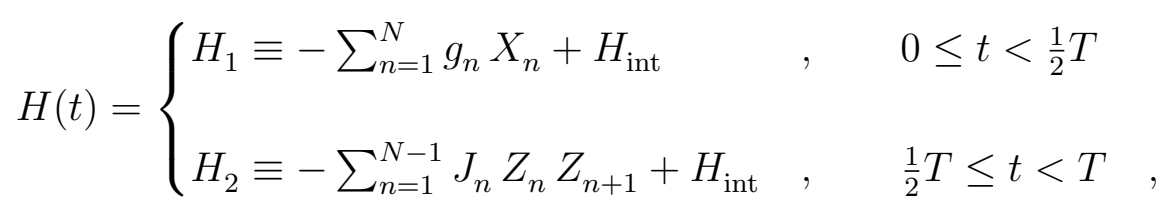

with $H(t+T)=H(t)$, and where $H_{\text {int }}=K \sum_{n=1}^{N-1} X_{n} X_{n+1}$ is an interaction Hamiltonian. The Floquet unitary is $U_{\mathrm{F}}=e^{-i H_{2} T / 2 \hbar} e^{-i H_{1} T / 2 \hbar}$. The transverse fields $g_{n}$ and the $Z Z$ Ising couplings $J_{n}$ are random at each location along the chain. When $K=0, U_{\mathrm{F}}$ can be expressed in terms of noninteracting fermions; this is the 'free fermion parent' of the KLMS time crystal. When $K>0$ the $X X$ interaction spoils this feature, but all the Floquet eigenstates still may be classified by their $X$-parity, $P_{X}=\prod_{n=1}^{N} X_{n}$. The phase diagram in Fig. 1a is shown in terms of the couplings $\bar{g}$ and $\bar{J}$, which for noninteracting fermions represent disorder averages. In the presence of interactions, these phases are robust ${ }^{2}$. The four phases are:

Paramagnet (PM): This is a symmetry-unbroken phase in which the spins are mainly polarized along the transverse field. The spin-spin correlation function $C_{l, l^{\prime}}^{\alpha}=\left\langle\phi_{\alpha}\left|Z_{l} Z_{l^{\prime}}\right| \phi_{\alpha}\right\rangle$ tends to zero at long distances in all the Floquet eigenstates.

SPT Paramagnet $(0 \pi \mathrm{PM})$ : The bulk spins again lie in the direction of the transverse field, and the bulk correlations $C_{l, l^{\prime}}^{\alpha}$ decay to zero at large distances; there is no spontaneous

\footnotetext{
${ }^{2}$ In the presence of Zeeman terms $h_{n} Z_{n}$ which break Ising $X$-parity, only the $\pi$ SG/DTC is absolutely stable, and the other three phases are adiabatically connected.
} 
symmetry breaking. The eigenspectrum of $U_{\mathrm{F}}$ is arranged into quartets, where the splittings are exponentially close to 0 and $\pi$, an effect due to boundary spin degeneracy. On a ring, there are no edges and all states are nondegenerate. This phase is thus a symmetry-protected topological phase (SPT), similar to the $S=1$ Haldane spin chain. In both cases, the edge physics can be elicited by making entanglement cuts on the ring. At the upper edge of the phase diagram, for homogeneous $J_{n}=\bar{J}$, the edge spins each get flipped once per cycle, so by continuity this phase may be considered a 'boundary DTC'. The protecting symmetry is Ising parity $P_{X}$ and so this phase is unstable with respect to the presence of Zeeman terms of the form $h_{n} Z_{n}$.

Spin Glass (0SG): Now the spins are polarized along $Z$, and $C_{l, l^{\prime}}^{\alpha}$ is nonzero. However, since $\bar{X}$-parity is still a good quantum number, each of the eigenstates of $U_{\mathrm{F}}$ is a superposition, á la Schrödinger's cat, of a state with spin-glass order and its spin-reversed mate, with the glassiness due to domain walls induced by the random transverse fields. These states are doubly degenerate in their quasienergies, up to exponentially small splittings, because each cat state is formed from a superposition of macroscopically distinct components. There are no cat states in the spectra of the thermal, PM and $0 \pi P M$ phases.

$\pi$-Spin Glass (Discrete Time Crystal) $(\pi \mathrm{SG} / \mathrm{DTC})$ : Once again $C_{l, l^{\prime}}^{\alpha}$ is nonzero at large separations, for all Floquet eigenstates. The cat states are no longer degenerate, but are separated in their quasienergies by $\frac{1}{2} \hbar \Omega$. This is related to the fact that, for uniform $g_{n}=\bar{g}$, along the line $\bar{g}=\frac{1}{2} \hbar \Omega$, one has $\exp \left(i H_{1} T / 2 \hbar\right)=i^{N} P_{X}$, which in the $Z$ basis flips each spin once per cycle, performing a perfect $\pi$-pulse. What is remarkable is that this behavior is stable with respect to disorder, and indeed to any weak period- $T$ perturbation. As emphasized in ref. [4], this absolute stability is a property only of the DTC, and by adding a longitudinal Zeeman term $\sum_{n} h_{n} Z_{n}$ in the Floquet drive, the $0 S G$ and $0 \pi \mathrm{PM}$ phases can be adiabatically connected to the trivial PM phase.

This picture has been confirmed by numerical simulations [2]. Very encouragingly, soon afterwards, experiments in which qubits were realized by spin impurities in room temperature diamond [10], and trapped atomic ions in a spin chain configuration [11], reported the observation of a long-lived period-doubled spin response ${ }^{3}$. It has since turned out that these systems did not realize eigenstate-ordered DTCs. Rather, they have served to enlarge our understanding of how thermalization takes place. Ref. [10] is now understood to be a case of slow thermalization across the entire spectrum due to the presence of long-ranged dipolar interactions, while ref. [11] is apparently a case of prethermalization [13], where heating is slow due to a mismatch between driving and internal energy scales, and the subharmonic generation is associated with initial states being drawn from a very small and special fraction of the total spectrum.

The theoretical requirements for the observation of an eigenstate-ordered DTC phase were elucidated in table 1 of ref. [14]. The significance of the Google AI experiment, which brings to bear many state-of-the-art tools from the field of quantum information, lies in meeting all of these.

The Google processor (see Fig. 2a) consists of a chain of qubits connected to gates which

\footnotetext{
${ }^{3}$ These experiments were in part inspired by the theoretical work of ref. [12], which proposed signatures such as imperfect spin echo as a protocol for identifying time crystalline behavior.
} 

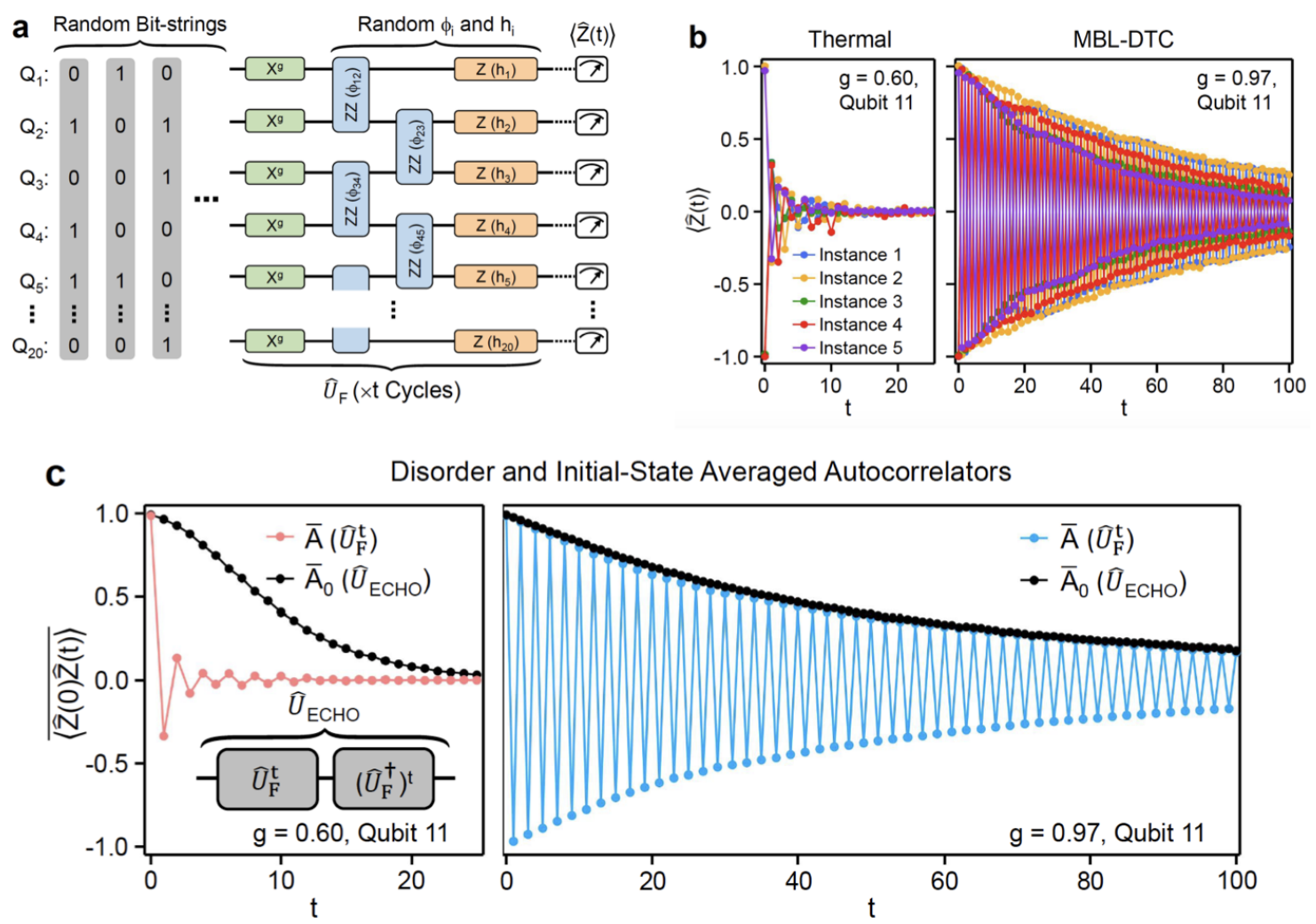

Figure 2: (a) A sketch of the quantum processor circuit which reifies the model of eqn. 2. (b) Measurements of the local qubit polarization $\left\langle Z_{n}(t)\right\rangle$ at $n=11$ in the Floquet-ETH phase (left) and the MBL-DTC phase (right), shown for five different configurations of the randomness. (c) The disorder-averaged autocorrelator $\overline{\mathrm{A}}(t)=\overline{\left\langle Z_{n}(t) Z_{n}(0)\right\rangle}$, again at $n=11$ and the echo $\overline{\mathrm{A}}_{0}(t)$ defined in the text. From ref. [6].

perform unitary operations. Such a device may be used to very naturally implement [14] Floquet drives. The experiment simulated, with $N=20$ transmon qubits, a model closely related to that in eqn. 1 , i.e.

$$
U_{\mathrm{F}}=\exp \left(-\frac{i}{2} \sum_{n=1}^{N} h_{n} Z_{n}\right) \exp \left(-\frac{i}{4} \sum_{n=1}^{N} \phi_{n} Z_{n} Z_{n+1}\right) \exp \left(-\frac{i}{2} \pi g \sum_{n=1}^{N} X_{n}\right)
$$

where $\left\{h_{n}\right\}$ and $\left\{\phi_{n}\right\}$ are random and positive, each with a bounded uniform distribution, with each $\phi_{n} \in\left[-\frac{3}{2} \pi .-\frac{1}{2} \pi\right]$ and $h_{n} \in[-\pi, \pi]$. Under special conditions, such as all $h_{n}=0$ or transverse field $g=1$, this model has certain discrete symmetries, such as $X$-parity or time-reversal symmetry. When $g \neq 1$ and $h_{n} \neq 0$, however, all discrete symmetries are broken. Nevertheless, the DTC is robust within a range of $g$ and $h_{n}$, due to its absolute stability $[3,4]$. The stability of the DTC phase even in the absence of $\mathbb{Z}_{2}$ spin flip symmetry was a key insight first provided by Else, Bauer, and Nayak [3]. As a (dynamical) phase of matter, the DTC is characterized by its spontaneous breaking of discrete time translation symmetry; it does not require the additional Ising $P_{X}$ symmetry in the model of eqn. 1 .

The circuit itself, which processes input bit-strings, is depicted in Fig. 2a. In the thermal phase, the spins thermalize at $T=\infty$, and $\left\langle Z_{n}(t)\right\rangle \rightarrow 0$, as confirmed in the first panel of Fig. 

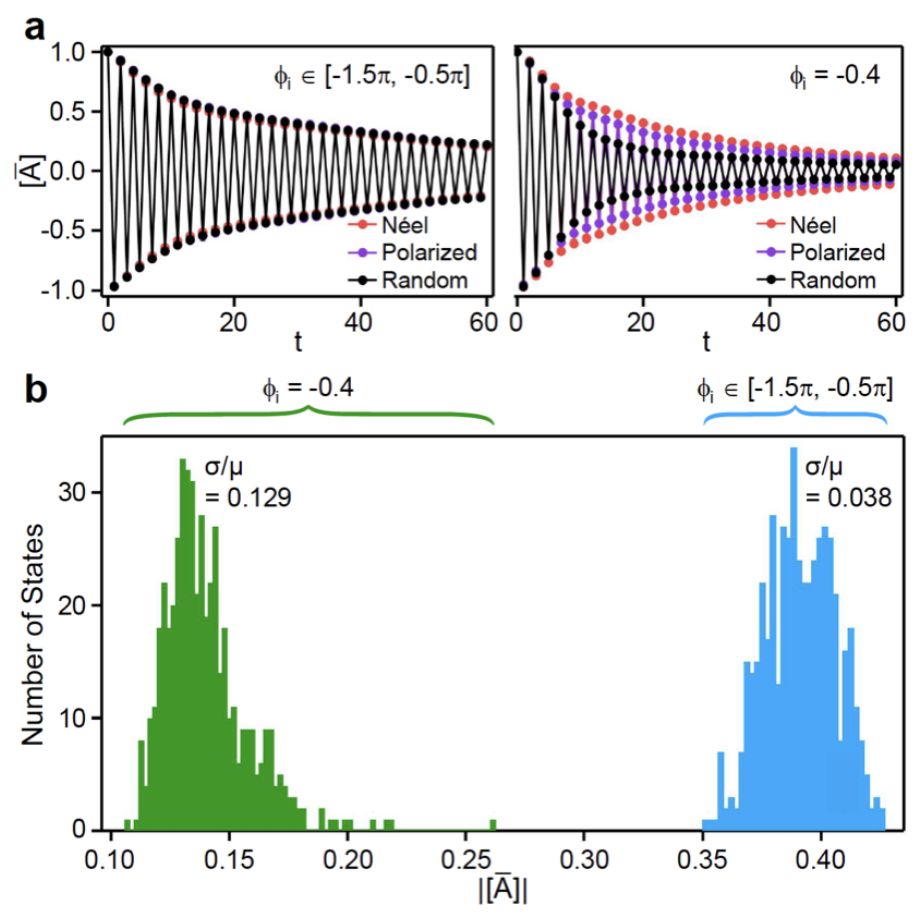

Figure 3: (a) Site- and disorder-averaged autocorrelator $[\overline{\mathrm{A}}(t)]$ for $g=0.94$ with disorder in $\left\{h_{n}, \phi_{n}\right\}$ (left) and in $\left\{h_{n}\right\}$ alone (right). In the latter case, the system is not in an MBL phase but rather exhibits transient DTC-like properties. Results for three initial bit-strings are shown. (b) Histograms of $|[\overline{\mathrm{A}}(t)]|$, with 500 random input bit-strings, averaged over the same set of 24 disorder realizations in (a) and over cycles $t=30$ and $t=31$. From ref. [6].

$2 \mathrm{~b}(g=0.60)$. For $g=0.97$, the system is in the MBL-DTC phase and $\left\langle Z_{n}(t)\right\rangle$ oscillates at the subharmonic frequency $\frac{1}{2} \Omega$, as shown in the right panel. The disorder-averaged autocorrelator, $\overline{\mathrm{A}}(n, t)=\overline{\left\langle Z_{n}(0) Z_{n}(t)\right\rangle}$ behaves similarly, as shown in the right panel of Fig. 2c. However, these correlations decay on a time scale $\tau \approx 80 T$, which could be due either to slow thermalization, meaning we are in fact not in a MBL phase, or decoherence of the qubits themselves. To assess which is the case, the team cleverly designed an 'echo' protocol which measures the autocorrelator $\mathrm{A}_{0}(n, t)=\left\langle Z_{n} U_{\mathrm{ECHO}}^{\dagger}(t) Z_{n} U_{\mathrm{ECHO}}(t)\right\rangle^{1 / 2}$ and its disorder average $\overline{\mathrm{A}}_{0}(n, t)$, where $U_{\mathrm{ECHO}}(t)=U_{\mathrm{F}}^{-t} U_{\mathrm{F}}^{t}$, i.e. $t$ applications of the Floquet unitary $U_{\mathrm{F}}$ followed by $t$ applications of its inverse $U_{\mathrm{F}}^{\dagger}$. With no qubit decoherence, $U_{\mathrm{ECHO}}(t)=1$ and thus $\overline{\mathrm{A}}_{0}(n, t)=1$, yet the envelope shown in the black dots of both panels of Fig. 2c shows a decay, which therefore must be due to decoherence. Furthermore, the decay of $\overline{\mathrm{A}}_{0}(n, t)$ perfectly matches that of $\overline{\mathrm{A}}(n, t)$ and the ratio $\overline{\mathrm{A}}(n, t) / \overline{\mathrm{A}}_{0}(n, t)$ evinces DTC order.

An essential aspect of the MBL-DTC phase is that the entire many-body spectrum exhibits the same late time correlations, and these characteristic features are thus independent of the initial state. This is illustrated in the left panel of Fig. 3a, which shows the $[\overline{\mathrm{A}}(t)]$, which is the average of $\overline{\mathrm{A}}(n, t)$ over both position and disorder, with three different bit-string inputs. In the DTC the same oscillations are observed independent of the initial state, which is consistent with the presence of eigenstate order. When the $\left\{\phi_{n}\right\}$ values are uniform (right panel), the system is no longer in an MBL phase. Histograms of the autocorrelator over 

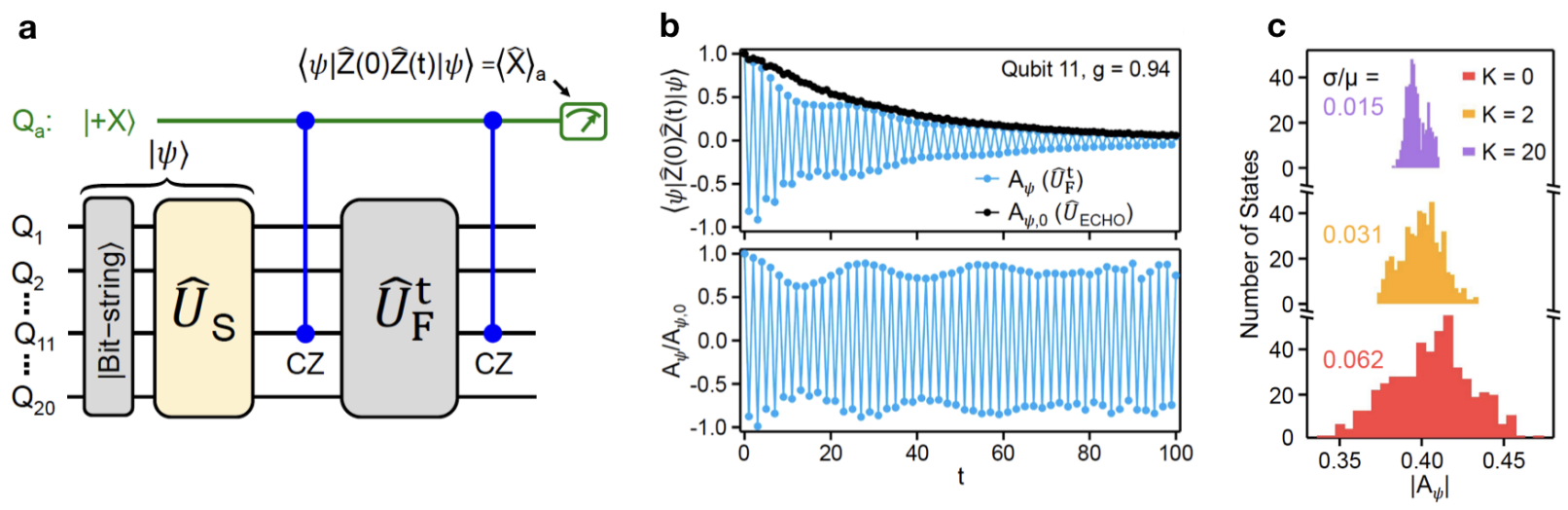

Figure 4: (a) Schematic for measuring the autocorrelator $A(n, t)=\left\langle Z_{n}(0) Z_{n}(t)\right\rangle$ where the input bit-string is scrambled by the operator $U_{\mathrm{S}}$, which implements $K$ layers of random single-qubit rotations interleaved with CZ gates. (b) $\mathrm{A}(n, t)$ with $K=20$ cycles in $U_{\mathrm{S}}$ for a single disorder realization (upper panel) and $n=11$, showing also the echo result (black curve), and the normalized correlator $\mathrm{A}(n, t) / \mathrm{A}_{0}(n, t)$ (bottom panel). (c) Histograms of distributions of $|\mathrm{A}(n, t)|$ averaged over cycles $t=30$ and $t=31$, again for a single disorder instance, and for different values of the layer number $K$. From [6].

cycles $t=30$ and 31 are shown in Fig. 3b, averaged over the same disorder but now 500 initial bit-string inputs. For the DTC phase, the ratio $\sigma / \mu$ of the standard deviation to the mean is $0.038,{ }^{4}$ while in the uniform $\phi_{n}$ phase the histogram is asymmetric and much broader in units of $\mu$, with $\sigma / \mu=0.129$.

Furthermore, by scrambling the input bit-string, one can effectively average over a vastly larger number of independent inputs, approaching an average over the entire many-body Hilbert space with the Haar invariant measure - 500 seems a goodly number of initial configurations, but $2^{20} \approx 10^{6}$ is much larger! Averaging over the entire Hilbert space with the Haar measure is, to exponential accuracy, equivalent to evaluating $\mathrm{A}(n, t)$ using a 'typical' initial state which is chosen from a Haar random distribution. Such an initial state is a random superposition over the $2^{N}$ basis states, which requires large resources to create. A schematic for how this is approximately achieved is depicted in Fig. 4a. An initial bitstring is subjected to a unitary scrambler $U_{\mathrm{S}}$ composed of $K$ alternating layers of random single-qubit rotations interleaved with quantum $\mathrm{CZ}$ gates ${ }^{5}$. This does not achieve true Haar randomness, but the greater the depth $K$, the closer one gets ${ }^{6}$.

\footnotetext{
${ }^{4}$ In the thermodynamic limit one should have $\sigma / \mu=0$, and the small but finite value is attributed to finite size and the relatively small number of disorder instances.

${ }^{5} \mathrm{~A} \mathrm{CZ}$ ('controlled $Z$ ') gate acts on the four-element two qubit basis as $\operatorname{diag}(1,1,1,-1)$. These gates entangle pairs of qubits.

${ }^{6} \mathrm{~A}$ truly Haar random initial state should give (to exponential accuracy) a zero width distribution, and for all correlators, but this would require an exponentially large depth of the scrambler. For $K=N=20$, a distribution of the scrambled states generates a '2-design', meaning that averages of all polynomials of degree $k=2$ in the local spin operators with respect to this distribution are guaranteed to coincide with their averages with respect to the full Haar measure. For a $k$-design distribution, averages of all polynomials of order $k$ in the local operators are equal to their averages with respect to the Haar measure. This affords a scalable protocol for computing spectral averages. Still, it is worth reiterating that the average here is with
} 

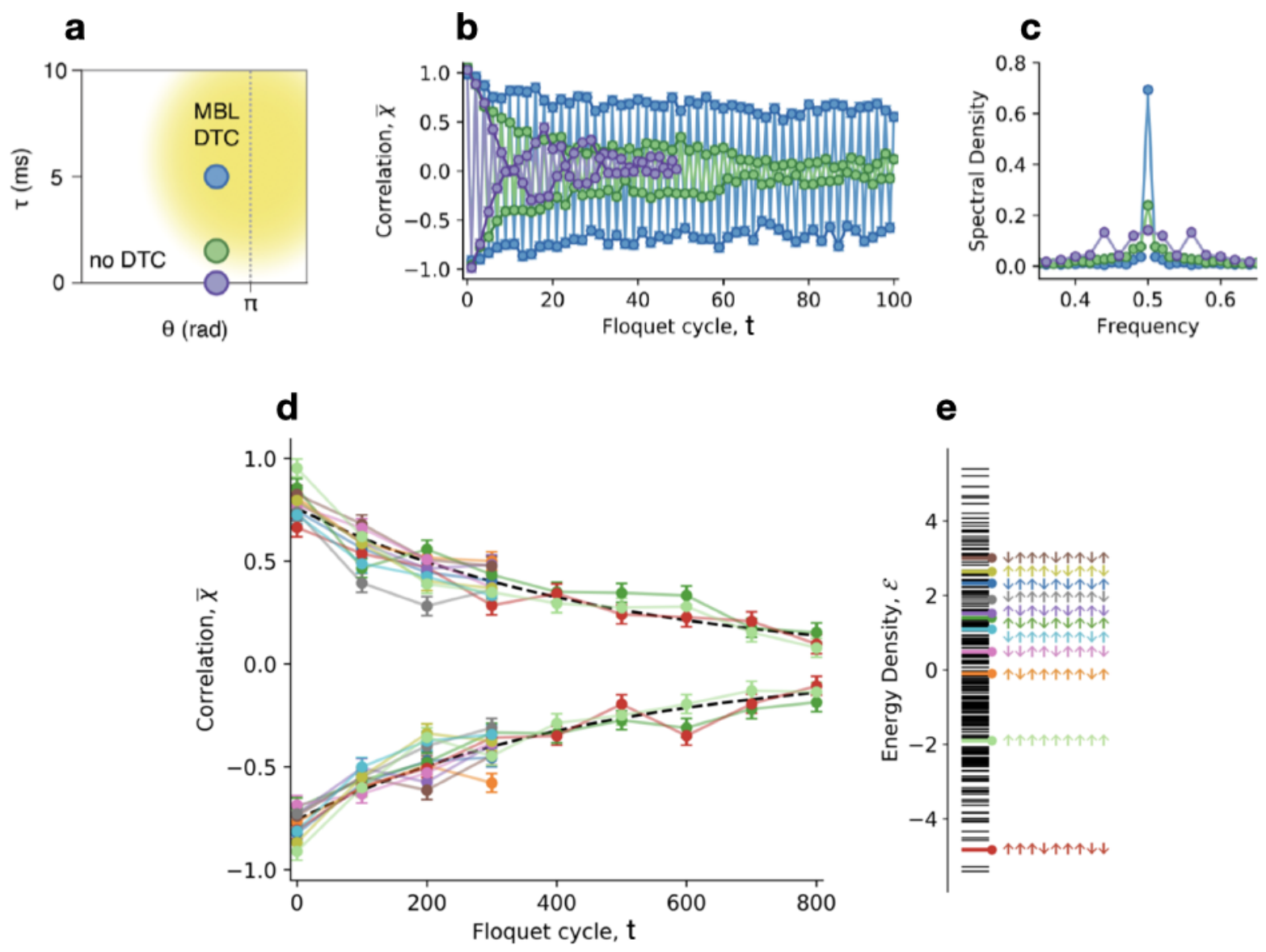

Figure 5: (a) Rough phase diagram in the $(\theta, \tau)$ plane. (b) Site-averaged autocorrelator $\bar{\chi}(t)$ for the three different locations in (a), with fully polarized initial state. (c) The Fourier transform of $\bar{\chi}(t)$ shows a sharp peak in the MBL-DTC phase. (d) $\bar{\chi}(t)$ in the MBL-DTC phase for even (upper) and odd (lower) cycle times for nine randomly chosen initial states. Three states are measured to $t=800$ and the remainder to $t=300$. Dashed black curves show the averages. (e) Energy density spectrum of all $2^{10}$ possible bit-string configurations in the MBL-DTC phase, evaluated with respect to an effective Hamiltonian derived from the leading order Floquet-Magnus expansion of $U_{\mathrm{F}}$. From ref. [7].

Fig. 4b (top panel) shows the behavior of the autocorrelator $\mathrm{A}(n, t)$ for a single disorder realization and a single initial state generated by a $K=20$ cycle unitary scrambler acting on an input bit-string. The corresponding echo result $\mathrm{A}_{0}(n, t)$ shown in black (top panel). The bottom panel shows the normalized autocorrelator $\mathrm{A}(n, t) / \mathrm{A}_{0}(n, t)$. Fig. 4c shows a histogram of those $|\mathrm{A}(n, t)|$ values averaged over cycles $t=30$ and 31 for different depths $K$ of the scrambler $U_{\mathrm{s}}$. What is found is that as $K$ increases, the width of the distribution gets progressively smaller, with the ratio $\sigma / \mu=0.015$ for $K=20$, which is considerably smaller than the value found by averaging over 500 input bit-strings and 24 disorder realizations. This is strong evidence for eigenstate ordering in the MBL-DTC phase.

respect to a single initial state, albeit a highly entangled one, and not the entire 2-design distribution. 
The Delft simulator's qubits are ${ }^{13} \mathrm{C}$ nuclear spins in diamond [7]. Although this work utilized not quite half as many qubits $(N=9)$ as that of the Google processor, the simulation times were substantially longer, reaching $t=800$ cycles as compared with Google's 100 . The simulator evolves its qubits according to the Floquet unitary $U_{\mathrm{F}}=U_{Z}(\tau) U_{X}(\theta) U_{Z}(\tau)$, where $U_{Z}(\tau)=\exp \left(-i H_{Z} \tau / \hbar\right)$, with

$$
H_{Z}=\sum_{n}\left(B+h_{n}\right) Z_{n}+\sum_{n<n^{\prime}} J_{n n^{\prime}} Z_{n} Z_{n^{\prime}}
$$

and $U_{X}(\theta)=\exp \left(-\frac{i}{2} \theta \sum_{n} X_{n}\right)$. Here $B$ is a magnetic field splitting, $h_{n}$ is a local frequency shift due to the electron-nuclear hyperfine interaction, and $J_{n n^{\prime}}$ are the $z z$-components of the magnetic dipole interaction between spins $n$ and $n^{\prime}$. The spins are arranged in a onedimensional chain, where MBL can be stabilized under periodic driving despite the presence of long-ranged interactions $J_{n n^{\prime}} \sim\left|n-n^{\prime}\right|^{-3}$. When $\theta=\pi$, the model is time-reversal symmetric, which is to say $\mathcal{T}_{X} U_{\mathrm{F}} \mathcal{T}_{X}^{-1}=U_{\mathrm{F}}^{\dagger}$, where $\mathcal{T}_{X}=K P_{X}$, with $K$ complex conjugation, is an (anti-unitary) time-reversal operator. Taking $\theta \neq 0, \pi$ breaks all discrete symmetries.

Fig. 5a shows a rough phase diagram in the $(\theta, \tau)$ plane for the model of eqn. 3. Panel (b) shows the autocorrelation function $\bar{\chi}(t)=N^{-1} \sum_{n=1}^{N}\left\langle Z_{j}(t)\right\rangle\left\langle Z_{j}(0)\right\rangle$ as a function of the Floquet cycle number $t$. The value of $\theta$ is fixed at $\theta=0.95 \pi$, which is to say deliberate underrotation, and the initial state is fully polarized $|\uparrow \uparrow \cdots \uparrow\rangle$. When $\tau=0$, one has $H_{Z}=0$ and the spins are consistently underrotated, leading to a decay in $\bar{\chi}(t)$. For $\tau=5 \mathrm{~ms}$, the system is in the MBL-DTC phase and $\bar{\chi}(t)$ decays slowly, and the envelope of the period $\Delta t=2$ oscillations loses half its amplitude over $\sim 100$ cycles. The discrete Fourier transform of $\bar{\chi}(t)$ is shown in panel (c), and exhibits a pronounced peak at the subharmonic frequency $\frac{1}{2}$ in the MBL-DTC phase. To test for dependence on initial conditions, the Delft group measured $\bar{\chi}(t)$ for nine different initial states of the form $\left|\sigma_{1} \cdots \sigma_{9}\right\rangle$, depicted in panel (d), with energy densities shown relative to the entire spectrum in panel (e) ${ }^{7}$.

The Delft results, for a single disorder realization and with nine spins, thus conform to the general desiderata of ref. [14], showing both subharmonic behavior in the autocorrelator $\bar{\chi}(t)$ which does not vanish upon spatial averaging, and which survives up to $t=800$ cycles, i.e. long-lived spatiotemporal response to the Floquet drive, as well as independence on initial conditions.

It should be noted that the very existence of MBL phases, even in $d=1$, is an increasingly murky issue. A recent study [15] has concluded that numerical work heretofore has vastly overestimated the stability of static MBL phases, and that the regime of true MBL behavior occurs at substantially weaker values of the interaction strength (at fixed disorder) than previously believed. While Google's processor seems impressively clean and scalable, both the Google and Delft simulators involved small numbers of qubits. Could it be that the Google simulator in fact exhibits a finite correlation time, even after decoherence effects are nulled out, which is simply longer than can presently be observed? The Google team also measured the disorder-averaged spin-glass order parameter $\chi^{\mathrm{SG}}$ as a function of the transverse field strength $g$, and performed a scaling analysis as a function of chain length in order to

\footnotetext{
${ }^{7}$ The energy densities were computed from $\left\langle\psi\left|H_{\text {eff }}\right| \psi\right\rangle / N$, where $H_{\text {eff }}$ is an effective Floquet Hamiltonian obtained by going to first nontrivial order in the expansion of $H_{\mathrm{F}}=i \hbar \tau^{-1} \log U_{\mathrm{F}}$.
} 
determine the critical point at which MBL-DCT order is lost, finding $0.83 \lesssim g_{\mathrm{c}} \lesssim 0.88$. While this was necessarily done over a finite range of cycle times (between $t=51$ and $t=60$ ), it would seem that at $g=0.97$ their system is well in the MBL phase.

The Google Quantum AI experiment in particular has set a standard in the verification of quantum dynamics. In identifying the possibility of multiple Floquet-MBL phases, the work of Khemani et al. opens the door to new possible dynamical phases of quantum matter stabilized by disorder and interactions, and indeed several new examples have already been identified theoretically. Rapid advances in quantum information device design and implementation are sure to provide us with additional checks on these and other fascinating theoretical ideas in the foreseeable future.

I am grateful to John McGreevy for helpful discussions.

\section{References}

[1] F. Wilczek, Phys. Rev. Lett. 109, 160401 (2012); H. Watanabe and M. Oshikawa, Phys. Rev. Lett. 114, 251603 (2015).

[2] V. Khemani, A. Lazarides, R. Moessner, and S. L. Sondhi, Phys. Rev. Lett. 116, 250401 (2016); R. Moessner and S. L. Sondhi, Nat. Phys. 13, 424 (2017).

[3] D. V. Else, B. Bauer, and C. Nayak, Phys. Rev. Lett. 117, 090402 (2016).

[4] C. W. von Keyserlingk, V. Khemani, and S. L. Sondhi, Phys. Rev. B 94, 085112 (2016).

[5] L. Balents, Jour. Club Condens. Matter, June 2016, No. 1.

[6] Google Quantum AI and collaborators, arXiv:2107.13571.

[7] J. Randall et al., arXiv:2107.00736.

[8] R. Nandkishore and D. A. Huse, Annu. Rev. Condens. Matter Phys. 6, 15 (2015).

[9] D. A. Huse, R. Nandkishore, V. Oganesyan, A. Pal, and S. L. Sondhi, Phys. Rev. B 88, 014206 (2013).

[10] S. Choi et al., Nature 543, 221 (2017).

[11] J. Zhang et al., Nature 543, 217 (2017).

[12] N. Y. Yao, A. C. Potter, I.-D. Potirniche, and A. Vishwanath, Phys. Rev. Lett. 118, 030401 (2017); Phys. Rev. Lett. 118, 269901 (2017).

[13] D. V. Else, B. Bauer, and C. Nayak, Phys. Rev. X 7, 011026 (2017).

[14] M. Ippoliti, K. Kechedzhi, R. Moessner, S. L. Sondhi, and V. Khemani, PRX Quantum 2, 030346 (2021).

[15] A. Morningstar, L. Colmenarez, V. Khemani, D. J. Luitz, and D. A. Huse, et al., arXiv:2107.05642. 